\title{
Multi Objective Optimization for Turning Operation using Hybrid Extreme Learning Machine and Multi Objective Genetic Algorithm
}

\author{
Tiagrajah V. Janahiraman ${ }^{1 *}$, Nooraziah Ahmad ${ }^{2}$ \\ ${ }^{I}$ Centre for Signal Processing and Control Systems, Dept. of Electronics and Communication Engineering, \\ College of Engineering, Universiti Tenaga Nasional, Jalan IKRAM-UNITEN, 43000 Kajang, Selangor, Malaysia \\ ${ }^{2}$ Faculty of Creative Technology and Heritage, Universiti Malaysia Kelantan, \\ Kampus Bachok, Locked bag No. 1, 15300 Bachok, Kelantan,Malaysia. \\ *Corresponding authorE-mail:tiagrajah@uniten.edu.my
}

\begin{abstract}
Turning operation, a type of machining process using Computer Numerical Control (CNC) machine in which a cutting tool, typically a non-rotary tool bit, moves to describe a helix toolpath while the cylindrical metal workpiece rotates. Numerous conflicting performance functions such as maximizing material removal rate, minimizing the product's quality, maximizing the tool life and others, remains crucial for a system to optimize in order to obtain optimum benefit. The machinist is required to assign the optimal cutting parameters in $\mathrm{CNC}$ turning machine which have direct influence on the performance of each cutting process and machined product. It is very crucial for optimal parameters selection to maximize the performance function. A new optimisation model has been proposed in this paper. This model, uses Box Behnken Design (BBD) for design of experiment and the prediction model has been developed using Extreme Learning Machine (ELM) which is tuned using Particle Swarm Optimization. A powerful and effective, Multi Objective Genetic Algorithm (MOGA) will act as an optimizer of the developed model. Turning input parameters such as feed rate, cutting speed and depth of cut were considered as input variables and surface roughness, specific power consumption and cutting force were used as output variables. This novel approach, BBD-ELM-PSO-MOGA can predict the optimal cutting parameters as demonstrated in our case studies with less number of tunable parameters and number of experiments. Therefore, it is fast, less time consuming and easy to be implemented.
\end{abstract}

Keywords: Turning operation, Multi objective Optimization Genetic Algorithm, Extreme Learning Machine, Box Behnken Design, Particle Swarm Optimization.

\section{Introduction}

Metal cutting has become a very large sector in Malaysia. Turning operation is one of the process which include straight cutting, taper turning, profiling, grooving, facing, threading and drilling. Conventional lathe, which is manipulated by operator and the computer controlled Computer Numerical Control (CNC) machine are two examples of lathe machines used in the industry. Since the introduction of $\mathrm{CNC}$ in manufacturing, metal machining companies prefer to use $\mathrm{CNC}$ rather than conventional lathe. Using $\mathrm{CNC}$, manufacturing cost can be significantly reduced, without compromising on quality of producing parts which are cut at high precision, accuracy and increased machine tool efficiency. In order to run a CNC machine, the machinist needs to enter the parameter values for the selected design and material.

These input values, which are referred as cutting parameters, will directly influence the performance(s) of the final product [1] There are three main cutting parameters in turning operation which are depth of cut, cutting speed and feed rate. Normally, the machinist will determine the parameters based on his/her working experience and use the manual handbook provided the machine manufacturer as a guide. Yet, these suggested values will not be the most accurate for optimal cutting parameters of a selected design and material.

In order to solve this issue, the statistical approaches such as Taguchi method (TM) and Response Surface Methodology were introduced by $[2,3]$. The researchers gave higher priority for TM, Asilturk and Akkus [3] used TM in finding the optimal cutting parameters for minimizing the surface roughness while Camposeco-Negrete [4] used it to minimize the energy consumption for turning operation. Yet, these conventional optimization approaches were unable to solve multi objective problem. Many multi objective performances involve conflicting performance function such as maximizing material removal rate, minimizing the product's quality (dimensional accuracy/ surface roughness), maximizing the tool life, and reducing the effect towards the environment and many more [5]. There are some researchers who have tried to solve multi objective problem using modified conventional optimization approaches. But their multi objective methods tend to converge into local minima based solution $[6,7,8]$. It becomes more complicated when many practical constraints were considered [7]. In order to achieve optimum values, the researchers conducted many experiments $[9,10]$.

Thus, the metaheuristic method is chosen to deal with the conventional optimization problem. The modified metaheuristic is intro- 
duced over normal algorithm such that it can give the nondominated solutions or Pareto optimal solution for multi objective function. The Pareto optimal is a set of solutions that consider all the objectives without sacrificing at least one objective function [11]. Santana et al. [12] used Particle Swarm Optimization (PSO) which has a diversity mechanism called crowding distance and roulette wheel to deal with multi objective optimization. Mandal et al. [13] has reported that Genetic Algorithm (GA) has widely used in determining optimal cutting parameters considering multi objectives. Among the approaches discussed, Non-dominated Sorting Genetic Algorithm 2 (NSGA-2) is identified to be the most powerful and fast approach. Many have proved that GA gives better result in optimizing the machining parameters for multi objective optimization problem $[7,14,15]$.

However, most of the literature on metaheuristic algorithm have predefined empirical models for their performances [7, 16]. But, none of them explained on how they propose the empirical model. Therefore, based on the reviews, improved optimization method is introduced in this paper to provide the whole process of optimization which starts from modelling up to solving the simultaneous multi objective optimization. The aim of this systematic approach is to deliver optimal cutting parameters for multi objective performances in turning operation with the lowest number of samples for modelling phase without compromising on accuracy. The rest of the paper is organized as follows: The proposed method will be explained in Section 2 while the experimental setup and cutting conditions are defined in Section 3. Section 4 discusses the results and finally, this paper is concluded in Section 5.

\section{Proposed Method}

The proposed optimization method is a combination of statistical and artificial intelligence (AI) techniques, as shown in Figure 1. It contains three stages which are preparation of experimental data, modelling and optimization. For the first stage, the statistical technique will be used to generate the data set for the modelling phase. In this stage, the lowest number of data set with the best prediction accuracy for the cutting performance will be identified. In the second stage, an AI model will used for training and prediction of the require performance. In the third stage, an optimizer will determine the optimal input by using the trained model.

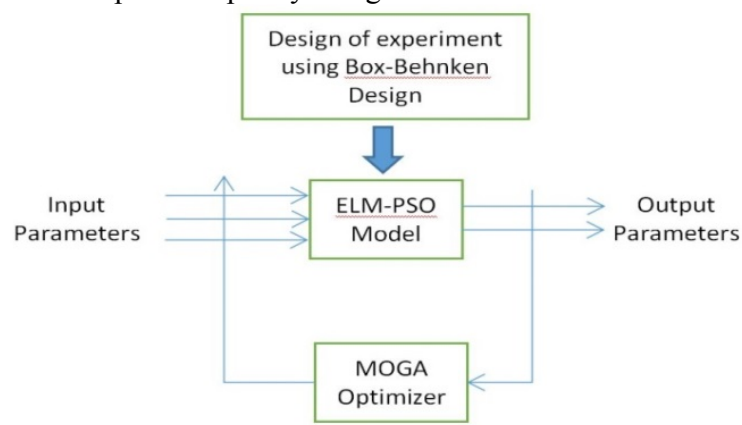

Fig. 1: Block diagram of the whole system

\subsection{Box Behnken Design (BBD)}

Design of Experiment (DOE) is a systematic approach which can be applied in many engineering problem. The Response Surface Methodology (RSM), a type of DOE, which can identify the relationship between variable and the performance(s) systematically. There are two types of RSM which are Central Composite design (CCD) and Box Behnken design (BBD). When compared to CCD, $\mathrm{BBD}$ requires least number of experiments as it does not consider the point at the vertices of the experiment region. The advantage using the midpoints of edges instead of vertices is the level combinations might be easy to test since it is inside the physical process constraint. The Figure 2 shows an example of geometric representation of three factors Box-Behnken design.

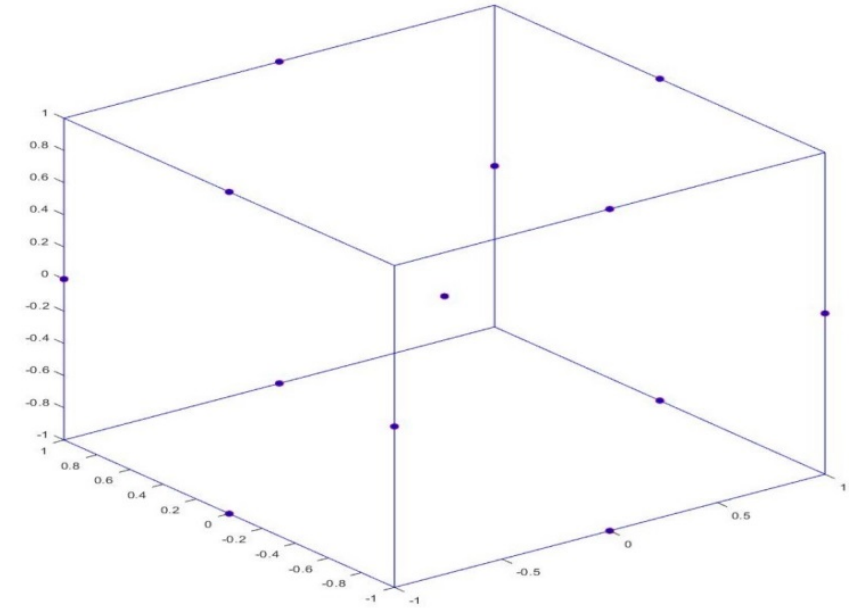

Fig. 2: Geometric representation of BBD for three factors

According to Ahmad et al, BBD with RSM proved to be the most reliable experimental design that could be used in predicting the performance function of machining [17]. The case study conducted by the authors show that for three levels and factors experiments, the BBD provided the smallest number of experiments, which is fifteen, and produced smaller average variance compared to others experimental designs. For this reason, BBD has been chosen as the experimental design in the modelling technique.

\subsection{Extreme Learning Machine (ELM)}

In 2006, Huang et al proposed a modeling technique called Extreme Learning Machine (ELM). The enhancement proposed by $\mathrm{Xu}$ and Shu proved to be the fastest and has good generalization achievement when compared to conventional ELM [18]. Each layer is connected by the weight $(w)$. During the modelling process, these weights are randomly assigned. It does not need to be tuned like the traditional single layer feed forward neural network but manage to develop an accurate model even though it has a limited data [19]. The ELM works by finding the hidden to output weights $(\widehat{\boldsymbol{\beta}})$, as shown in Equation 1, using Moore-Penrose pseudo inverse matrix provided by Equation 2 .

\section{$\widehat{\boldsymbol{\beta}}=\boldsymbol{H}^{+} \boldsymbol{T}$}

where $H$ is the hidden output and $H^{\dagger}$ is the Moore-Penrose generalized inverse of matrix $H$.

$H=\left[\begin{array}{ccc}f\left(w_{1} x_{1}+b_{1}\right) & \cdots & f\left(w_{N} x_{1}+b_{N}\right) \\ \vdots & \ddots & \vdots \\ f\left(w_{1} x_{M}+b_{1}\right) & \cdots & f\left(w_{N} x_{M}+b_{N}\right)\end{array}\right]$

and $T$ is the target, $T=\left(T_{1}, \ldots, T_{\mathrm{N}}\right)^{\mathrm{T}}$.

The ELM is considered to reach the solution when it has achieved the properties in Equation 3 and 4.

1. The trained error for ELM to reach the minimum value.

$\min \|\boldsymbol{H} \widehat{\boldsymbol{\beta}}-\boldsymbol{T}\|$

2. The output weight for the ELM is the smallest weight.

$\min \|\widehat{\boldsymbol{\beta}}\|$

The prediction result often ends up being unsatisfactory because the ELM input weight and hidden bias are always chosen randomly. The author in [20] discusses the improved variant of ELM, where the weights from input layer to hidden layer were optimized using Particle Swarm Optimization (PSO). Experimental results in [20] shows that improved ELM can produce the best performance based on the ELM architecture. The advantage of combining ELM 
with PSO includes the fact that only minimum parameter is needed to be adjusted. It also considers a particle taken from the real number while decreasing the norm of the output weights. Besides that, the input weight and hidden biases are constrained within a appropriate range in order to improve the ELM performance. Further details on the modelling method have been explained in detail in [20]. The parameters for our ELM architecture are given in Table 1 .

Table 1: Parameters for ELM

\begin{tabular}{|l|c|}
\hline Parameters & Description \\
\hline Number of input nodes & 3 \\
\hline Number of hidden nodes & 11 \\
\hline Number of output nodes & 2 \\
\hline Activation function at hidden layer & Tangent Hyperbolic \\
\hline Activation function at output layer & Linear \\
\hline
\end{tabular}

\subsection{Particle Swarm Optimization (PSO)}

Kennedy and Eberhart [21] introduced PSO to find input weight and bias which could fit prediction model in optimal manner. The swarms refer to the population while the potential solution refers to the particle inside PSO. The velocity calculation of each particle is shown in equation 5 and 6 . The particle will be improved based on its local best position within its neighbourhood and also the global best position according to the whole swarm population.

$$
v_{i}^{k+1}=w v_{i}^{k}+c_{1} R_{1}\left(p_{i}-x_{i}^{k}\right)+c_{2} R_{2}\left(p_{g}-x_{i}^{k}\right)
$$

$x_{i}^{k+1}=x_{i}^{k}+v_{i}^{k+1}$

where $w$ is the inertia weight, while $c_{1}$ and $c_{2}$ refers to the local and global acceleration coefficients, respectively with the interval of $0<c_{1} ; c_{2}<2$. In this study, the input weights and biases are randomly assigned and will be the PSOs' particles. Each particle will has its own fitness value which is the error on the validation data set. This error will be calculated based on Box Behnken Design - Extreme Learning Machine - Particle Swarm Optimization (BBDELM-PSO). Then, the particle will improve its position and velocity by iteratively modifying its personal best position $p_{1}$ and the global best position $p_{g}$. The $c_{1}$ and $c_{2}$ are set to be 2 while $w$ is equal to 1 . To achieve the minimum prediction error of the test data set and the smallest norm of output weight, the process of adjustment of $p_{l}$ and $p_{g}$ will be repeated. The result achieved for this algorithm will be the input weight and bias which has the lowest validation error among its population.

\subsection{Multi Objective Genetic Algorithm (MOGA)}

Multi objective optimization makes its decision by evaluating the given mathematical model of more than one constraint at the same time. In our context, the output parameters such as cutting force, surface roughness and specific power consumption need to be minimized. In the final stage, Multi Objective Genetic Algorithm (MOGA), is selected as the optimizer. Therefore, the overall optimization method proposed by the author is BBD-ELM-PSOMOGA. The MOGA design was implemented using MATLAB software. MOGA was run with default parameters set in the toolbox, as summarized in Table 2 .

Table 2: MOGA Parameter details

Table 2: MOGA Parameter details
\begin{tabular}{|l|c|}
\hline Parameters & Details \\
\hline Population size & 30 \\
\hline Crossover rate & 0.75 \\
\hline Mutation type & Polynomial \\
\hline Termination criterion & 700 \\
\hline
\end{tabular}


Table 7: Testing value for prediction model

\begin{tabular}{|c|c|c|c|}
\hline $\begin{array}{c}\text { Measured surface rough- } \\
\text { ness (SR) }(\mu \mathrm{m}) \text { by Nayak } \\
\text { et al.(2014) }\end{array}$ & $\begin{array}{c}\text { Predicted } \\
\text { SR }(\mu \mathrm{m})\end{array}$ & $\begin{array}{c}\text { Measured } \\
\text { force }(\mathrm{N}) \text { by } \\
\text { Nayak et al. } \\
(2014)\end{array}$ & $\begin{array}{c}\text { Predicted } \\
\text { force }(\mathrm{N})\end{array}$ \\
\hline 0.49 & 0.41 & 476 & 478 \\
\hline 0.57 & 0.60 & 839 & 839 \\
\hline 0.44 & 0.42 & 860 & 858 \\
\hline
\end{tabular}

Table 8: The predicted optimal cutting parameters

\begin{tabular}{|l|c|c|}
\hline & $\begin{array}{c}\text { Nayak et al. } \\
(2014)\end{array}$ & $\begin{array}{c}\text { Proposed } \\
\text { method }\end{array}$ \\
\hline Cutting speed $(\mathrm{m} / \mathrm{min})$ & 35 & 40 \\
\hline Feed rate $(\mathrm{mm} / \mathrm{rev})$ & 0.1 & 0.1 \\
\hline Depth of cut $(\mathrm{mm})$ & 1.5 & 1.5 \\
\hline
\end{tabular}

\section{Discussion}

Based on the experimental parameters for 15 experiments that were generated using BBD, our modelling and prediction were performed using the ELM-PSO architecture. The corresponding outputs of our model were compared with each case study, Ramanujam et al [9] and Nayak et al [10], by using MAE given in equation 7. The results tabulated in Table 9 shows that MAE between our proposed model and the two case studies, as shown in Table 4 and 7 , are less than 0.1 for all performances. The nearer to zero error indicates that the develop model can predict the performances very well. An important point to note here is our proposed method was able to produce almost the same output value as the other two case studies with smaller number of experiments, which gives a significant reduction in the cost of conducting experiments.

Table 9: Mean absolute error for modelling and prediction stage

\begin{tabular}{|c|c|c|}
\hline Case study & Performance & MAE \\
\hline \multirow{2}{*}{ Ramanujam et al. (2011)[9] } & Surface roughness & 0.02 \\
\cline { 2 - 3 } & Power & 0.08 \\
\hline \multirow{2}{*}{ Nayak et al. (2014)[10] } & Surface roughness & 0.02 \\
\cline { 2 - 3 } & Force & 0.04 \\
\hline
\end{tabular}

\section{Conclusion}

In this study, BBD-ELM-PSO-MOGA approach are proposed to optimize the cutting parameters for surface roughness in the turning operation. The machinist will never need to assume the cutting parameters or running the trial and error experiment as the method can give proper way in finding the optimal using BBD. The proposed modelling method is fast in training the parameters. The method is also proved to be accurate and it requires less adjusting parameters.

The MOGA produced an estimated value which is very close to the true optimal solution for multi objective performances. This proposed approach is fast, less time consuming and easy to implement due to the less parameter required. For the purpose of future work, this approach can be applied for the optimisation of other machining process and the creation of an interface for easy user interaction.

\section{References}

[1] D. Baji'c, L. Celent, and S. Jozi'c, "Modeling of the influence of cutting parameters on the surface roughness, tool wear and the cutting force in face milling in off-line process control," Strojniski vestnik-Journal of Mechanical Engineering, vol. 58, no. 11, p. 673 , 2012.

[2] S. Nes, eli, S. Yaldız, and E. T"urkes, "Optimization of tool geometry parameters for turning operations based on the response surface methodology," Measurement, vol. 44, no. 3, pp. 580-587, 2011.

[3] I. Asilt"urk and H. Akkus, "Determining the effect of cutting parameters on surface roughness in hard turning using the taguchi method," Measurement, vol. 44, no. 9, pp. 1697-1704, 2011.
[4] C. Camposeco-Negrete, "Optimization of cutting parameters for minimizing energy consumption in turning of aisi 6061 t6 using taguchi methodology and anova," Journal of Cleaner Production, vol. 53, pp. 195-203, 2013.

[5] I. Mukherjee and P. K. Ray, "A review of optimization techniques in metal cutting processes," Computers \& Industrial Engineering, vol. 50, no. $1-2$, pp. $15-34,2006$.

[6] I. Asilt"urk and S. Nes, eli, "Multi response optimisation of cnc turning parameters via taguchi method-based response surface analysis," Measurement, vol. 45, no. 4, pp. 785-794, 2012.

[7] S. Yang and U. Natarajan, "Multi-objective optimization of cutting parameters in turning process using differential evolution and nondominated sorting genetic algorithm-ii approaches," The International Journal of Advanced Manufacturing Technology, vol. 49, no. 5-8, pp. 773-784, 2010.

[8] S. Xie and Y. Guo, "Intelligent selection of machining parameters in multi-pass turnings using a ga-based approach," Journal of Computational Information Systems, vol. 7, no. 5, pp. 1714-1721, 2011.

[9] R. Ramanujam, N. Muthukrishnan, and R. Raju, "Optimization of cutting parameters for turning al-sic (10p) mmc using anova and grey relational analysis," International Journal of Precision Engineering and Manufacturing, vol. 12, no. 4, pp. 651-656, 2011.

[10] S. K. Nayak, J. K. Patro, S. Dewangan, and S. Gangopadhyay, Multiobjective optimization of machining parameters during dry turning of aisi 304 austenitic stainless steel using grey relational analysis," Procedia Materials Science, vol. 6, pp. 701-708, 2014.

[11] M. Solimanpur and F. Ranjdoostfard, "Optimisation of cutting parameters using a multi-objective genetic algorithm," International Journal of Production Research, vol. 47, no. 21, pp. 6019-6036, 2009.

[12] R. A. Santana, M. R. Pontes, and C. J. Bastos-Filho, "A multiple objective particle swarm optimization approach using crowding distance and roulette wheel," in Intelligent Systems Design and Applications, 2009. ISDA'09. Ninth International Conference on, pp. 237-242, IEEE, 2009.

[13] D. Mandal, S. K. Pal, and P. Saha, "Modeling of electrical discharge machining process using back propagation neural network and multiobjective optimization using non-dominating sorting genetic algorithm ii," Journal of Materials Processing Technology, vol 186, no. 1-3, pp. 154-162, 2007.

[14] Y. Yusoff, M. S. Ngadiman, and A. M. Zain, "Overview of nsga-ii for optimizing machining process parameters," Procedia Engineering, vol. 15, pp. 3978-3983, 2011.

[15] S. Kuriakose and M. Shunmugam, "Multi-objective optimization of wire-electro discharge machining process by non-dominated sorting genetic algorithm," Journal of materials processing technology, vol. 170, no. 1-2, pp. 133-141, 2005.

[16] R. Q. Sardinas, M. R. Santana, and E. A. Brindis, "Genetic algorithm based multi-objective optimization of cutting parameters in turning processes," Engineering Applications of Artificial Intelligence, vol. 19, no. 2, pp. 127-133, 2006.

[17] N. Ahmad and T. V. Janahiraman, "A study on regression model using response surface methodology," Applied Mechanics and Materials, vol. 666, pp. 235-239, 2014.

[18] Y. Xu and Y. Shu, "Evolutionary extreme learning machine--based on particle swarm optimization," in International Symposium on Neural Networks, pp. 644-652, Springer, 2006.

[19] C. Natarajan, S. Muthu, and P. Karuppuswamy, "Prediction and analysis of surface roughness characteristics of a non-ferrous material using ann in cnc turning," The International Journal of Advanced Manufacturing Technology, vol. 57, no. 9-12, pp. 10431051, 2011.

[20] N. Ahmad and T. V. Janahiraman, "Modelling and prediction of surface roughness and power consumption using parallel extreme learning machine based particle swarm optimization," in Proceedings of ELM-2014 Volume 2, pp. 321-329, Springer, 2015.

[21] J. Kennedy and R. Eberhart, "Particle swarm optimization. proc. ieee international conference on neural networks (perth australia)," in IEEE Service Center, Piscataway, NJ, IV, pp. 1942-1948. 